\title{
Anti-Proliferative and Pro-Apoptotic Activities of Hederacolchiside A1 On MCF-7 Cells Via ROS Regulation and JAK2/STAT3 Inactivation
}

\section{Aijun Chen}

Zhejiang University

Shushu Zhang

Soochow University

Dandan Zhang

Shenyang Pharmaceutical University

Xingjiang Hu

Zhejiang University

Nana Xu

Zhejiang University

Jichen Li

Zhejiang University

Qiao Zhang

Zhejiang University

Jincai Lu

Shenyang Pharmaceutical University

Xia Wu ( $\nabla$ wuxia_michelle@zju.edu.cn )

Zhejiang University

Original article

Keywords: Hederacolchiside A1, MCF-7, Apoptosis, ROS, JAK2/STAT3

Posted Date: April 14th, 2021

DOl: https://doi.org/10.21203/rs.3.rs-408362/v1

License: (c) (1) This work is licensed under a Creative Commons Attribution 4.0 International License.

Read Full License 


\section{Abstract}

Many studies have shown that hederacolchiside A1 (HA1) is an important anticancer saponin, although its mechanism of action and in vivo investigations are still lacking. Our previous results revealed that HA1 may have the potential to treat breast cancer. Therefore, we attempted to verify the potential anti-breast cancer effect of HA1 in vitro and in vivo. MTT, flow cytometry, DCFH-DA fluorescence microscopy, and western blotting were used to evaluate the activities and mechanisms of action of HA1. Athymic nude mice were used to demonstrate the antitumor activity of HA1 in vivo. HA1 exhibited significant cytotoxic effects on HepG2, MCF-7, MDA-MB-231, SKBr-3, HT-29, and HCT-116 cells, especially MCF-7 cells. HA1 blocked the sub-G1 and G0/G1 phases, induced apoptosis, promoted reactive oxygen species (ROS) generation, and decreased the mitochondrial membrane potential of MCF-7 cells. HA1 upregulated Bax and downregulated Bcl-2 levels and activated caspase- 9 and caspase-3 in MCF-7 cells Meanwhile, HA1 inhibited the phosphorylation of JAK2/STAT3 in MCF-7 cells. In addition, 50 and $100 \mathrm{mg} / \mathrm{kg} \mathrm{HA} 1$ significantly inhibited the growth of transplanted tumors with inhibition rates of $46.95 \pm 26.72 \%$ and $48.45 \pm 22.36 \%$, respectively. This preliminary study demonstrated that HA1 could inhibit proliferation and induce the apoptosis of MCF-7 cells via ROS-mediated activation of the mitochondrial apoptotic pathway and JAK2/STAT3 inactivation. HA1 may therefore be developed as a novel agent for breast cancer therapy.

\section{Key Points}

1. We systematically summarized all the results about the antiproliferative activity of HA1 for the first time and further enriched them.

2. This is a further confirmatory research on the anti-breast tumor active of HA1 on MCF-7 cells in vitro and in vivo.

3. HA1 was firstly reported to induce the apoptosis of MCF-7 cells by inducing ROS generation and inactivating JAK2/STAT3 pathway.

\section{Introduction}

Breast cancer is the most common malignant tumor in women globally and is the main cause of cancerassociated deaths in women (Park et al. 2020). The anticancer active ingredients isolated from Traditional Chinese Medicine (TCM) have shown good efficacy after validation via extensive experiments and clinical studies (Wang et al. 2021). The signal transducer and activator of transcription 3 (STAT3) signaling pathway, which can be activated by Janus kinase (JAK), was found to be strongly related to numerous tumorigenesis processes, including cell proliferation, sensitivity to cytotoxic agents, apoptosis, and cell death (Sansone et al. 2012). The activation of the JAK2/STAT3 signaling pathway could promote tumor growth by affecting the expression of cell cycle regulatory and antiapoptotic genes, and STAT3 inhibitors may exhibit more promising effects on breast cancer therapy (Ma et al. 2020). 


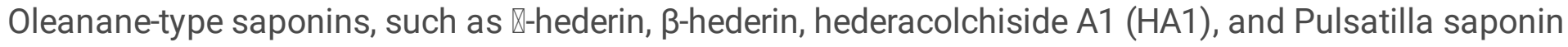
$A / D$, have received considerable attention because of their strong antitumor activity in vitro and in vivo (Wang et al. 2017; Guan et al. 2020). HA1, as the main active ingredient, widely exists in the genera Anemone L. and Hedera L. These include Pulsatilla chinensis (Bunge) Regel, Anemone raddeana Regel, and Anemone flaccida Fr. Schmidt. HA1 and its derivatives reportedly exhibit strong antitumor activities against various cell lines in vitro, with $\mathrm{IC}_{50}$ values of less than $10 \mu \mathrm{M}$ (Li et al. 2018; Liang et al. 2016; Fang et al. 2016). Although the anticancer potential of HA1 has been extensively reported, investigations of its antitumor activity in vivo and the mechanism of action are lacking.

Our previous results showed that HA1 exhibited strong cytotoxicity in a variety of cancer cell lines with $\mathrm{IC}_{50}$ values of 0.29-3.48 $\mu \mathrm{M}$ (Zhao et al. 2018). We also reported that TSS (the alkaline hydrolysis product of $A$. raddeana) showed excellent antitumor activity against breast cancer both in vitro and in vivo. HA1 is the main active ingredient and mainly contributes to the anticancer effect of TSS (Zhang et al. 2020). In addition, previous studies have reported that the growth and metastasis of 4T1 cell (mouse breast cancer cells) xenografts could be inhibited by $5 \mathrm{mg} / \mathrm{kg} \mathrm{HA} 1$ after intraperitoneal injection (Liang et al. 2016). These data suggest that HA1 might play a crucial role in the proliferation and progression of breast cancer, although these results are still inconclusive. As a part of our continued investigation of HA1 (Zhang et al. 2017a; Zhang et al. 2020), this study first attempted to summarize the anticancer effects of HA1 in vitro and in vivo. Next, we investigated the mechanism of the antiproliferation and proapoptotic activities of HA1 on MCF-7 cells. Moreover, the antitumor effect of HA1 was further confirmed in the MCF-7 xenograft tumor model.

\section{Materials And Methods}

\section{Antibodies and reagents}

Antibodies against Bcl-2, Bax, caspase-3/9, cleaved caspase-3/9, and the internal reference $\beta$-actin were purchased from Beyotime Institute of Biotechnology (Jiangsu, China). Antibodies against JAK2, phosphorylated-JAK2 (p-JAK2), STAT3, and phosphorylated-STAT3 (p-STAT3) were obtained from Cell Signaling Technology Inc. Other reagents and test kits, such as N-acetyl-L-cysteine (NAC), Rhodamine123 (Rh123), Cell Cycle Detection Kit, Apoptosis Detection Kit, and reactive oxygen species (ROS) detection kits were purchased from Beyotime (Shanghai, China). As a continuation of our previous work, HA1 was isolated and purified from A. raddeana (Zhao et al. 2018; Zhang et al. 2020).

\section{Cell cytotoxicity assay}

As we previously reported, HepG2, MCF-7, MDA-MB-231, SKBr-3, HT-29, and HCT-116 cells were purchased from the Shanghai Institute of Cell Resource Center Life Science. The cytotoxicity of HA1 was assessed by an MTT staining assay in 96-well plates. Several different dosages of HA1 $(0.625,1.25,2.5,5,10,20$, and $40 \mu \mathrm{M})$ and the control group (0.1\% DMSO) were coincubated for $48 \mathrm{~h}$.

\section{Cell cycle detection}


The cell cycle was detected as previously reported without any change (Zhang, 2020). After treatment with HA1 $(4,8$, and $12 \mu \mathrm{M})$ for $24 \mathrm{~h}$, the cells were digested, collected, centrifuged, washed, and fixed overnight. After centrifugation and washing with phosphate-buffered saline (PBS), $100 \mu \mathrm{L}$ RNase A was added to MCF-7 cells. Then, the cells were incubated at $37^{\circ} \mathrm{C}$ for $30 \mathrm{~min}$, and $5 \mu \mathrm{L} \mathrm{PI} \mathrm{was} \mathrm{added} \mathrm{and}$ mixed. Finally, the DNA content was detected using the same flow cytometer that we used previously (Zhang et al. 2020).

\section{Annexin V-FITC/PI double staining assay}

MCF-7 cells were inoculated in 6-well plates and exposed to different dosages of HA1 $(4,8$, and $12 \mu \mathrm{M})$ for $48 \mathrm{~h}$. Then, following the manufacturer's instructions, cells $\left(1 \times 10^{5}\right)$ were collected, centrifuged, and washed with PBS. PI and Annexin V-FITC ( $5 \mu \mathrm{L}$ each) staining were then performed room temperature for 15 min in the dark. Finally, the cells were analyzed using a flow cytometer.

\section{ROS generation detection}

Following the manufacturer's instructions, ROS generation in MCF-7 cells after treatment with HA1 was detected by fluorescence staining using 2',7'-dihydrofluorescein-diacetate (DCFH-DA). Cells were first incubated with fresh medium containing $10 \mu \mathrm{M}$ DCFH-DA for $20 \mathrm{~min}$ in the dark in 6-well plates. The cells were then exposed to different concentrations of HA1 $(4,8$, and $12 \mu \mathrm{M})$ for $30 \mathrm{~min}$ in an incubator. Finally, after washing with PBS three times, the cells were photographed using a fluorescence microscope (Olympus, Tokyo).

\section{Mitochondrial membrane potential (MMP) detection}

MMP was detected using Rhodamine 123 dye, as previously described (Zhang et al. 2015). After treatment with HA1 for $24 \mathrm{~h}$, the MCF-7 cells were washed twice with PBS. Then, the cells were stained with Rhodamine $123(1 \mathrm{mM})$ and incubated for $30 \mathrm{~min}$. Finally, cells with fluorescence were photographed using the same fluorescence microscope (Olympus, Tokyo, Japan).

\section{Western blot analysis}

Following a previously established protocol (Zhang et al. 2017b), MCF-7 cells were collected and lysed in RIPA buffer (containing 10\% PMSF) after incubation with HA1 for $48 \mathrm{~h}$. Total protein was quantified with the BCA protein assay kit after centrifugation according to the manufacturer's instructions. Then, $40 \mu \mathrm{g}$ total protein was loaded onto 10\% SDS-PAGE and separated by electrophoresis. After isolation, the proteins were transferred to PVDF membranes. The membranes were blocked with $5 \%$ blocking buffer and incubated with Bcl-2, Bax, caspase-3/9, cleaved caspase-3/9, JAK2/STAT3, and $\beta$-actin at appropriate dilutions overnight. Secondary antibodies were incubated for $2 \mathrm{~h}$, and the membranes were washed three times the next day. The chemiluminescence method was used to detect the protein content following the manufacturer's instructions (Beyotime, Jiangsu, China).

\section{In vivo antitumor activity}


BALB/c-nude female mice (16-20 g) were loaded subcutaneously with MCF-7 cells $\left(0.1 \mathrm{~mL}\right.$ of $5 \times 10^{6}$ cells/mouse) into the flank. After 6 days of tumorigenesis, 18 mice were randomly chosen and divided into three groups with six mice per group: the control group was treated with saline $(10 \mathrm{~mL} / \mathrm{kg} / \mathrm{mouse} / \mathrm{d})$ and the HA1 groups were administered HA1 (50 and $100 \mathrm{mg} / \mathrm{kg} / \mathrm{mouse} / \mathrm{d}$ ) for 28 days. After all mice were sacrificed, all tumors were obtained and measured. The tumor volume and inhibition rate (IR) of tumor weight were calculated.

\section{Statistical analysis}

All data are expressed as the mean \pm standard deviation (SD) of three experiments using GraphPad Prism software. One-way analysis of variance (ANOVA) with Dunnett's post-hoc test was used to compare the significant differences $(p<0.05)$ between groups.

\section{Results}

\section{HA1 exhibited cytotoxic effects on several cancer cell lines}

As summarized in Table S1, HA1 exhibited significant antitumor activities against dozens of cell lines in vitro, and the $\mathrm{IC}_{50}$ values were less than $10 \mu \mathrm{M}$. In this study, we evaluated the cytotoxicity of HA1 using an MTT assay on six cell lines (HepG2, MCF-7, MDA-MB-231, SKBr-3, HT-29, and HCT-116). As shown in Table 1, HA1 exhibited significant inhibitory activity against these cells $\left(\mathrm{IC}_{50}<10 \mu \mathrm{M}\right)$. HA1 exhibited the strongest antiproliferative effect on MCF-7 cells, with IC 50 values of $4.90 \pm 0.50 \mu \mathrm{M}$.

Table 1. Cytotoxic activity of HA1 on several cancer cell lines.

\begin{tabular}{|lllllll|}
\hline Cells & HepG2 & MCF-7 & MDA-MB-231 & SKBr-3 & HT-29 & HCT-116 \\
\hline $\mathrm{IC}_{50}$ values & $5.71 \pm 0.47$ & $4.90 \pm 0.50$ & $7.31 \pm 1.52$ & $8.67 \pm 1.37$ & $9.77 \pm 2.69$ & $8.93 \pm 1.77$ \\
$(\mu \mathrm{M})$ & & & & & & \\
\hline
\end{tabular}

\section{HA1 inhibited MCF-7 cell proliferation}

The structure of HA1 is shown in Fig. 1A. As shown in Fig. 1B, HA1 significantly inhibited the cell viability of MCF-7 cells when the dose was higher than $2.50 \mu \mathrm{M}$. The effect was difficult to observe when the HA1 dose was less than $1.25 \mu \mathrm{M}$; however, the cytotoxic activity was too high when the dosage was higher than $15 \mu \mathrm{M}$. We concluded that the effective dose range for HA1 was narrow. After constant exploration, HA1 dosages of 4,8 , and $12 \mu \mathrm{M}$ were chosen for the follow-up experiments in vitro.

\section{HA1 induced cell cycle arrest in MCF-7 cells}

The cell content in the sub-G1 and G0/G1 phases increased significantly when the dosage of HA1 was 12 $\mu \mathrm{M}$, while the $\mathrm{S}$ phase tended to decline. Meanwhile, HA1 induced the arrest of the sub-G1 phase in MCF7 cells after $24 \mathrm{~h}$ of treatment (Fig. $2 \mathrm{~A}$ ). These findings suggest that HA1 significantly blocked the cell 
cycle at the sub-G1 and G0/G1 phases, thus affecting DNA replication and inhibiting MCF-7 cell proliferation (Fig. 2B).

\section{HA1 induced MCF-7 cell apoptosis}

HA1 significantly triggered the apoptotic response and increased the percentage of apoptotic cells after $48 \mathrm{~h}$ of treatment, as detected by flow cytometry (Fig. 3A). The apoptosis rates of MCF-7 cells treated with different dosages of $\mathrm{HA} 1(4,8$, and $12 \mu \mathrm{M})$ were $17.94 \pm 4.75 \%, 31.80 \pm 5.75 \%$, and $97.10 \pm 10.43 \%$, respectively (Fig. 3B). These results preliminarily confirmed the proapoptotic effect of HA1.

\section{HA1 promoted ROS production and reduced MMP in MCF-7 cells}

HA1 (8 and $12 \mu \mathrm{M})$ could significantly increase the levels of intracellular ROS in MCF-7 cells from 101.23 $\pm 2.05 \%$ to $207.93 \pm 21.75 \%$ and $264.32 \pm 32.58 \%$, respectively, after 30 min incubation. However, NAC significantly decreased the ROS production to $103.27 \pm 23.76 \%$ (Fig. 4A and C). Considering that the proapoptotic activity was mediated by ROS, excessive ROS might have a large impact on the integrity of MMP. We detected the changes in MMP of MCF-7 cells after treatment with HA1 (8 and $12 \mu \mathrm{M}$ ) for $24 \mathrm{~h}$. The results showed that the fluorescence intensity of MCF-7 cells was significantly reduced by 8 and 12 $\mu \mathrm{M}$ HA1 from $99.57 \pm 3.75 \%$ to $68.51 \pm 11.09 \%$ and $52.63 \pm 5.37 \%$, respectively (Fig. 4B and D).

\section{HA1 induced MCF-7 cell apoptosis through the mitochondrial pathway}

The results of western blot analysis revealed that HA1 $(4,8$, and $12 \mu \mathrm{M})$ caused a significant decrease in the levels of caspase-3/9 and a remarkable increase of cleaved caspase-3/9 after $48 \mathrm{~h}$ of treatment. HA1 also caused a significant decline in Bcl-2 levels and increased Bax levels (Fig. 5A), which caused a significant increase in the ratio of Bax/Bcl-2 ratio after HA1 treatment (Fig. 5B). These results preliminarily confirmed that HA1 induced apoptosis in MCF-7 cells by activating the mitochondrial apoptotic pathway.

\section{HA1 inactivated the JAK2/STAT3 signaling pathway}

After treating cells with HA1 $(4,8$, and $12 \mu \mathrm{M})$ for $48 \mathrm{~h}$, the expression levels of JAK2, STAT3, p-JAK2, and p-STAT3 were determined. The levels of total JAK2 and STAT3 did not significantly change compared to the control; however, the levels of p-JAK2 and p-STAT3 were significantly reduced (Fig. 6A). HA1 (8 and 12 $\mu \mathrm{M})$ significantly inhibited the phosphorylation ratio (Fig. 6B). These results suggest that HA1 could inhibit the activation of the JAK2/STAT3 pathway.

\section{HA1 exhibit antitumor effects on mice}

To further confirm the antitumor effect of HA1 in vivo, tumor-bearing nude mice were subjected to experiments. At the end of the experiments on day 28 , HA1 (50 and $100 \mathrm{mg} / \mathrm{kg}$ ) significantly inhibited tumor growth in these tumor-bearing nude mice (Fig. 7A). The volume (Fig. 7B) and weight of tumors (Fig. 7C) of the 50 and $100 \mathrm{mg} / \mathrm{kg} \mathrm{HA} 1$-treated groups were significantly inhibited compared to those of 
the control group. The tumor volume decreased from $1,446.77 \pm 216.72 \mathrm{~mm}^{3}$ to $661.36 \pm 120.65 \mathrm{~mm}^{3}$ and $588.45 \pm 191.32 \mathrm{~mm}^{3}$ in the 50 and $100 \mathrm{mg} / \mathrm{kg} \mathrm{HA} 1$ groups, respectively, and the tumor weight decreased to $43.36 \pm 26.72 \%$ and $48.45 \pm 22.36 \%$ of the control group .

\section{Discussion}

Triterpenoid saponins, such as $\alpha / \beta / \delta$-hederin, hederagenin, hederacosides $A-I$, and hederacolchisides $(A$, $A 1$, and $B)$, are the main active phytochemical components that exhibit various biological activities.

These activities include cytotoxic, antiproliferative, antitumor, antimicrobial, antifungal, anthelmintic, and antileishmanial effects (Gumushan-Aktas et al. 2016; Wang et al. 2017). As shown in Table S1, previous studies have reported the cytotoxic activity of HA1 on numerous cell lines with $\mathrm{IC}_{50}$ values lower than 10 $\mu \mathrm{M}$. Our previous results showed that HA1 exhibited strong cytotoxicity in a variety of cell lines with $\mathrm{IC}_{50}$ values of $0.29-3.48 \mu \mathrm{M}$ (Zhao et al. 2018). HA1 has also been reported to exhibit significant inhibitory effects on breast cancer proliferation, invasion, and metastasis in vitro and in vivo (Liang et al. 2016; Wang et al. 2018). Similar to these results, this study further confirmed that HA1 exhibited a significant inhibitory effect on HepG2, MCF-7, MDA-MB-231, SKBr-3, HT-29, and HCT-116 cells and superior antiproliferative ability in MCF-7 cells.

As previously mentioned, cell cycle arrest is an effective measure to inhibit the abnormal proliferation of tumor cells (Zhang et al. 2020). A previous study reported that HA1 exhibited a significant inhibitory effect on the proliferation, invasion, and metastasis of many types of cancer cell lines by inducing cell cycle arrest (Debiton et al. 2004; Gerkens et al. 2007; Liang et al. 2016). Among them, HA1 could inhibit proliferation and induce apoptosis by arresting the cell cycle at the G0/G1 phase, accompanied by a decrease in cyclin D1, cyclin A, cyclin E, and p21 and p27 (Liang et al. 2016). Similar to these results, we found that HA1 inhibited the proliferation of MCF-7 cells by blocking the sub-G1 and G0/G1 phases. Therefore, HA1 may affect the mitosis of MCF-7 cells, thus terminating the normal operation of the cell cycle and inhibiting tumor cell proliferation.

Apoptosis is accompanied by changes in cell morphology, such as cell volume reduction, nuclear membrane shrinkage, chromatin concentration and aggregation, and apoptotic bodies (Zhang et al. 2015). HA1 has a significant inhibitory effect on many types of tumor cell lines (NCl-H460, ZR75-1, HL-60, SMMC-7721, Bel-7402, MCF-7) by inducing apoptosis (Gerkens et al. 2007; Liang et al. 2016; Wang et al. 2018; Guan et al. 2020). HA1 can reportedly induce the apoptosis of breast cancer cells (ZR75-1 and MCF-7) and increase the expression of cleaved PARP and caspase-3 (Liang et al. 2016). In addition, one of the derivatives of HA1 can induce cell apoptosis via mitochondria-mediated intrinsic pathways in HepG2 cells (Li et al. 2018). Our results showed that HA1 could induce the concentration and aggregation of chromatin, cause cell shrinkage, and induce the production of apoptotic bodies in MCF-7 cells. Meanwhile, HA1 significantly increased the percentage of apoptotic cells. These results preliminarily confirmed the proapoptotic effect of HA1. 
In addition, in our previous study, TSS with HA1 as the main active component did not affect the expression of Fas, suggesting that TSS may not induce MCF-7 cell apoptosis through an exogenous signaling pathway (Zhang et al. 2020). We further confirmed that HA1 could significantly downregulate $\mathrm{Bcl}-2$ and pro-caspase-3/9, as well as upregulate the expression of Bax and cleaved caspase-3. These results indicate that the caspase cascade reaction was triggered by HA1, which eventually led to the apoptosis of MCF-7 cells. This may be related to the effects of increased ROS levels and decreased MMP. These results suggest that the induction of apoptosis may be one of the mechanisms underlying the antiproliferative effect of HA1.

The molecular mechanisms of the antitumor effects of HA1 remain uncertain, except for its reported membrane damage and high affinity for melanin (Delmas et al. 2000; Debiton et al. 2004). To date, research on the molecular mechanism is mainly limited to the inhibition of the PI3K/AKT/mTOR signaling pathway (Wang et al. 2018; Guan et al. 2020; Zhang et al. 2020). It has been shown that STAT3 is crucial in numerous tumorigenesis processes, and activation of the JAK-STAT pathway plays a crucial role in the antiproliferation and apoptosis induction of breast cancer cells (Sansone et al. 2012; Ma et al. 2020). Similar saponin compounds, such as raddeanin A and a-hederin, have been reported to inhibit the JAK2/STAT3 pathway (Sun et al. 2018; Wang et al. 2019). Similarly, our results revealed that HA1 inhibited the phosphorylation of both JAK2 and STAT3. These results revealed that the inhibitory effect of JAK2/STAT3 pathway by HA1 may be one of its mechanisms of action.

Although many studies have indicated that HA1 has high antiproliferative activity in vitro, research on its antitumor effects in vivo is still lacking. HA1 has been reported to exhibit a potent antitumor effect in H22 xenografts (Fang et al. 2016a; Fang et al. 2017; Wang et al. 2018). Most in vivo studies have focused on anti-breast cancer; for example, the growth and metastasis of $4 \mathrm{~T} 1$ cell xenografts were significantly inhibited after intraperitoneal injection with HA1 (5 mg/kg) (Liang et al. 2016). HA1 (15 mg/kg) exerted an inhibitory effect on transplanted MCF-7 cells in nude mice (Wang et al. 2018). We also reported that HA1 was the main active ingredient of TSS (the alkaline hydrolysis product of $A$. raddeana), and TSS showed excellent antitumor activity against breast cancer both in vitro and in vivo (Zhang et al. 2020). Similar to these results, we preliminarily confirmed that HA1 (50 and $100 \mathrm{mg} / \mathrm{kg}$ ) could inhibit the growth of transplanted MCF-7 cells in vivo. These results reveal that HA1 may possess therapeutic potential in breast cancer. The difference in the tumor inhibition rate between 50 and $100 \mathrm{mg} / \mathrm{kg} \mathrm{HA} 1$ was not obvious; thus, in comparison to the dosage used in other studies (Fang et al. 2016; Fang et al. 2017; Liang et al. 2016; Wang et al. 2018; Kang et al. 2018), our dosage may have been too high. The dosage was set below $50 \mathrm{mg} / \mathrm{kg}$ in further experiments.

In conclusion, this study further confirmed that HA1 was cytotoxic to HepG2, MCF-7, MDA-MB-231, SKBr3, HT-29, and HCT-116 cells. HA1 significantly inhibited the proliferation of MCF-7 cells by promoting cell cycle arrest and inducing apoptosis, which may be mediated by promoting ROS generation, decreasing MMP, and activating the mitochondrial apoptosis pathway. Meanwhile, HA1 inhibits the activation of the JAK2/STAT3 pathway, which may contribute to the apoptosis-inducing effect of HA1. Finally, the 
antitumor activity of HA1 was confirmed in vivo. These results suggest that HA1 has the potential to be developed as a new agent for breast cancer therapy.

\section{Abbreviations}

DCFH-DA, 20,70-dichlorofluorescin diacetate; DMSO, Dimethyl sulfoxide; HepG2, Human hepatic carcinoma cells; JAK, Janus kinase; MCF-7, Michigan Cancer Foundation-7 breast cancer cells; MTT, 3(4,5-dimethylthiazol-2-yl)-2,5-diphenyl-tetrazolium bromide; NAC, N-acetyl---cysteine; PBS, Phosphate buffer saline; PI, Propidium iodide; Rh123, Rhodamine123; ROS, Reactive oxygen species; STAT3, Signal transducer and activator of transcription 3; TCM, Traditional Chinese Medicine; TSS, the alkaline hydrolysis product of Anemone raddeana Regel.

\section{Declarations}

\section{Acknowledgments}

We would like to thank Editage (www.editage.cn) for English language editing.

\section{Authors' contributions}

AC assisted the lead contributors to conduct the experiments, summarize the literatures and revise the manuscript. SZ and DZ contributed in designing and conducting the experiments, collecting the data, etc. $\mathrm{XH}, \mathrm{NX}, \mathrm{JL}$ and QZ provided technical guidance and modified the manuscript. JL, as the corresponding author, provided financial support. XW contributed in organizing, writing, editing and revising the manuscript.

\section{Fundings}

This work was supported by LiaoNing Revitalization Talents Program (No. XLYC1902119), National Science and Technology Major Project of the Ministry of Science and Technology of China (No. 2018ZX09735005), National Natural Science Foundation of China (81903849), Provincial Research Foundation of Zhejiang Integrative Association (Grant No. 2012LY018), Medical Health Science and Technology Project of Zhejiang Provincial Health Commission (2019RC034) and National Major Scientific and Technological Special Project for Significant New Drugs Development during the Thirteenth Five-year Plan Period (2020ZX 09201-003).

\section{Availability of data and materials}

All data generated or analysed during this study are included in this published article and its Additional files 1 .

\section{Ethics approval and consent to participate}


The animal experiment was approved by the Research Ethics Committee of Shenyang Pharmaceutical University (SYPU-IACUC-S2017-06.26-103).

\section{Consent for publication}

All authors approved the final version of the manuscript and consent for publication.

\section{Competing interests}

All authors declare that there's no conflict of interest.

\section{References}

Debiton, E., Borel, M., Communal, Y., Mshvildadze, V., Barthomeuf, C., 2004. In addition to membrane injury, an affinity for melanin might be involved in the high sensitivity of human melanoma cells to hederacolchiside A1. Melanoma Res. 14, 97-105.

Delmas, F., Di Giorgio, C., Elias, R., Gasquet, M., Azas, N., Mshvildadze, V., Dekanosidze, G., Kemertelidze, E., Timon-David, P., 2000. Antileishmanial activity of three saponins isolated from ivy, alpha-hederin, betahederin and hederacolchiside A1, as compared to their action on mammalian cells cultured in vitro. Planta Med. 66, 343-347.

Fang, Y., Wang, R., He, M., Huang, H., Wang, Q., Yang, Z., Li, Y., Yang, S., Jin, Y., 2017. Nitric oxide-donating derivatives of hederacolchiside A: Synthesis and biological evaluation in vitro and in vivo as potential anticancer agents. Bioorg. Med. Chem. Lett. 27, 98-101.

Li, H.N., Wang, H., Wang, Z.P., Yan, H.N., Zhang, M., Liu, Y., Cheng, M.S., 2018. Synthesis, antitumor activity evaluation and mechanistic study of novel hederacolchiside $A$ derivatives bearing an aryl triazole moiety. Bioorg. Med. Chem. 26, 4025-4033.

Gerkens, P.C., Dobson, R., Tabatadze, N., Mshviladzade, V., Elias, R., Peulen, O.J., Jolois, O.M., De, PauwGillet, M.C., 2007. Apoptosis and cytolysis induced by giganteosides and hederacolchisides in HL-60 cells. Anticancer Res. 27, 2529-2534.

Guan, Z., Chen, L., Zhou, Y., Luo, Y., Cui, Y., Liu, R., Shou, B., 2020. The synergistic antitumour effect of multi-components from Pulsatilla chinensis saponins in $\mathrm{NCl}-\mathrm{H} 460$ lung cancer cell line through induction of apoptosis. Pharm. Biol. 58, 427-437.

Gumushan-Aktas Hatice., Altun Seyhan. 2016. Effects of Hedera helix L. extracts on rat prostate cancer cell proliferation and motility. Oncol. Lett. 12(4), 2985-2991.

Kang, N., Shen, W., Gao, H., Feng, Y., Zhu, W., Yang, S., Liu, Y., Xu, Q., Yu, D., 2018. Antischistosomal Properties of Hederacolchiside A1 Isolated from Pulsatilla chinensis. Molecules 23, 1431.

Li, H.N., Wang, H., Wang, Z.P., Yan, H.N., Zhang, M., Liu, Y., Cheng, M.S., 2018. Synthesis, antitumor activity evaluation and mechanistic study of novel hederacolchiside A derivatives bearing an aryl triazole moiety. 
Bioorg. Med. Chem. 26, 4025-4033.

Liang, Y., Xu, X., Yu, H., L,i L., Hong, T., Ji, Q., Feng, Y., Jin, S., Song, Y., Guo, J., Zheng, Z., Ye, Q., Yang, S., 2016. Raddeanoside R13 inhibits breast cancer cell proliferation, invasion, and metastasis. Tumour Biol. 37, 9837-9847.

Ma J.H., Qin L., Li X., 2020. Role of STAT3 signaling pathway in breast cancer. Cell Commun. Signal 18: 33.

Park, H.S., Han, J.H., Park, J.W., Lee, D.H., Jang, K.W., Lee, M., Heo, K.Sun., Myung, C.S., 2020. Sodium propionate exerts anticancer effect in mice bearing breast cancer cell xenograft by regulating JAK2/STAT3/ROS/p38 MAPK signaling. Acta Pharmacol. Sin. undefined, undefined.

Sansone, P., Bromberg, J., 2012. Targeting the interleukin-6/Jak/stat pathway in human malignancies. J. Clin. Oncol. 30, 1005-1014.

Sun D., Shen W., Zhang F., Fan H., Xu C., Li L., Tan J., Miao Y., Zhang H., Yang Y., Cheng H., 2018. a-Hederin inhibits interleukin 6-induced epithelial-to-mesenchymal transition associated with disruption of JAK2/STAT3 signaling in colon cancer cells. Biomed. Pharmacother. 101, 107-114.

Wang, K., Chen, Q., Shao, Y., Yin, S., Liu, C., Liu, Y., Wang, R., Wang, T., Qiu, Y., Yu, H., 2021. Anticancer activities of TCM and their active components against tumor metastasis. Biomed. Pharmacother. 133, 111044.

Wang, L., Wang, Z., Su, S., Xing, Y., Li, Y., Li, M., Liu, J., Yang, S., 2017. Synthesis and cytotoxicity of oleanolic acid trisaccharide saponins. Carbohydr. Res. 442, 9-16.

Wang, Y.E., Xu, K., Yue, W.H., Xu, Q.M., You, B.G., Zhang, M.Y., Zhu, Z.C., Yang, S.L., Liu, Y.L., Li, K.P., 2018. Hederacolchiside $\mathrm{A} 1$ suppresses proliferation of tumor cells by inducing apoptosis through modulating PI3K/Akt/m TOR signaling pathway. Chin. Herb. Med. 10, 215-222.

Wang, Z., Shen, J., Sun, W., Zhang, T., Zuo, D., Wang, H., Wang, G., Xu, J., Yin, F., Mao, M., Zhou, Z., Hua, Y., Cai, Z., 2019. Antitumor activity of Raddeanin A is mediated by Jun amino-terminal kinase activation and signal transducer and activator of transcription 3 inhibition in human osteosarcoma. Cancer Sci. 110, 1746-1759.

Zhang, D., Lei, T., Lv, C., Zhao, H., Xu, H., Lu, J., 2017a. Pharmacokinetic studies of active triterpenoid saponins and the total secondary saponin from Anemone raddeana Regel. J. Chromatogr. B Analyt. Technol. Biomed. Life Sci. null, 54-62.

Zhang, D., Zhang, Q., Zheng, Y., Lu, J., 2020. Anti-breast cancer and toxicity studies of total secondary saponin from Anemone raddeana Rhizome on MCF-7 cells via ROS generation and PI3K/AKT/mTOR inactivation. J. Ethnopharmacol. 259, 112984. 
Zhang, Q., Cui, C., Chen, C.Q., Hu, X.L., Liu, Y.H., Fan, Y.H., Meng, W.H., Zhao, Q.C., 2015. Anti-proliferative and pro-apoptotic activities of Alpinia oxyphylla on HepG2 cells through ROS-mediated signaling pathway. J. Ethnopharmacol. 169, 99-108.

Zhang, Q., Hu, X., Hui, F., Song, Q., Cui, C., Wang, C., Zhao, Q., 2017b. Ethanol extract and its dichloromethane fraction of Alpinia oxyphylla Miquel exhibited hepatoprotective effects against $\mathrm{CCl}_{4}{ }^{-}$ induced oxidative damage in vitro and in vivo with the involvement of Nrf2. Biomed. Pharmacother. 91, 812-822.

Zhao, Y., Zhang, X., Lv, C., Yu, Y., Zhang, Y., Lu, J., 2018. Quantitative and qualitative analyses of cytotoxic triterpenoids in the rhizomes of Anemone raddeana using HPLC and HPLC-ESI-Q/TOF-MS. J. Food Drug Anal. 26, 1113-1121.

\section{Figures}




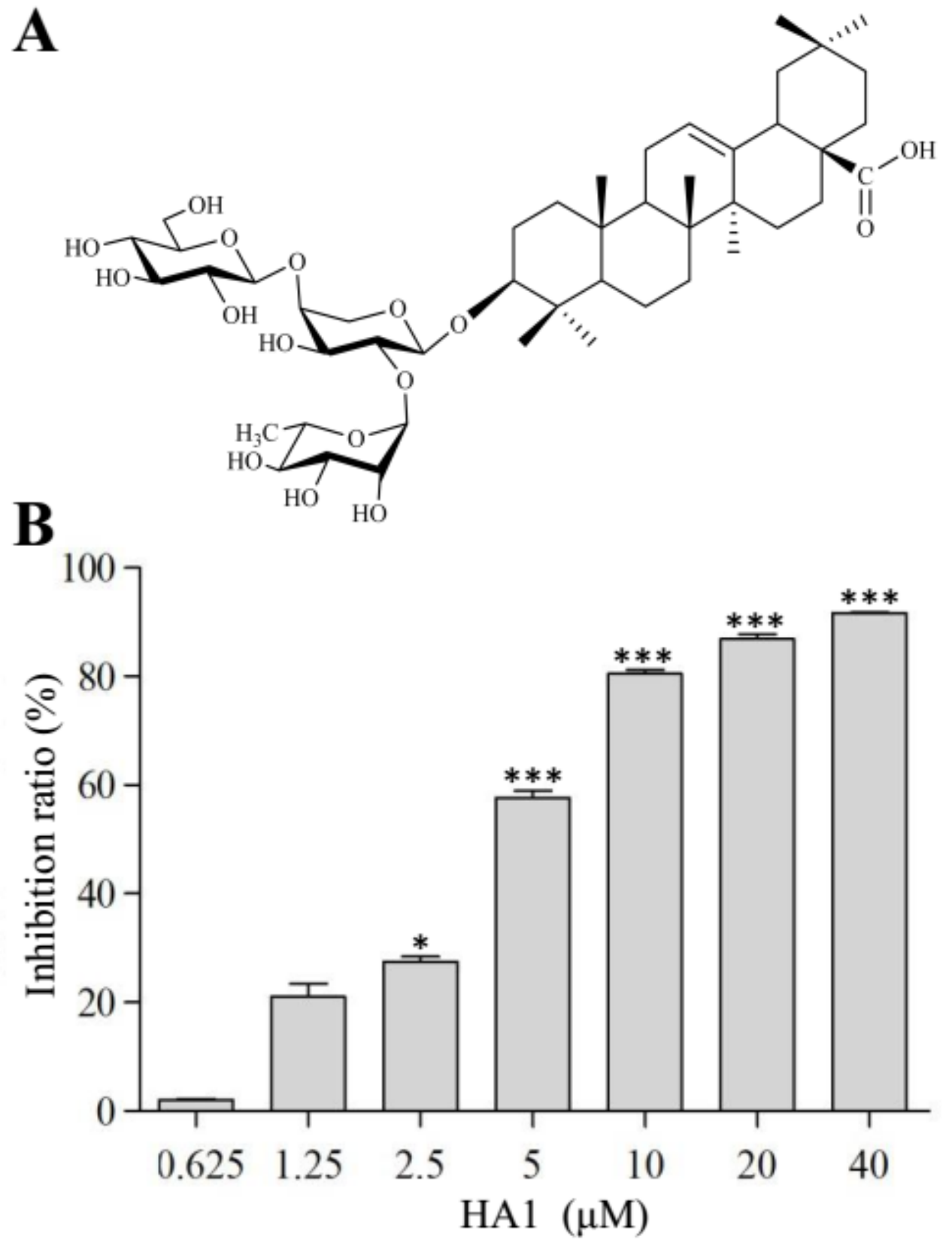

Figure 1

HA1 inhibit the proliferation of MCF-7 cells in a dosage dependent manner after $48 \mathrm{~h}$ treatment. *P $<0.05$ vs. Control; ${ }^{\star * \star P} \mathrm{P}<0.001$ vs. Control. 

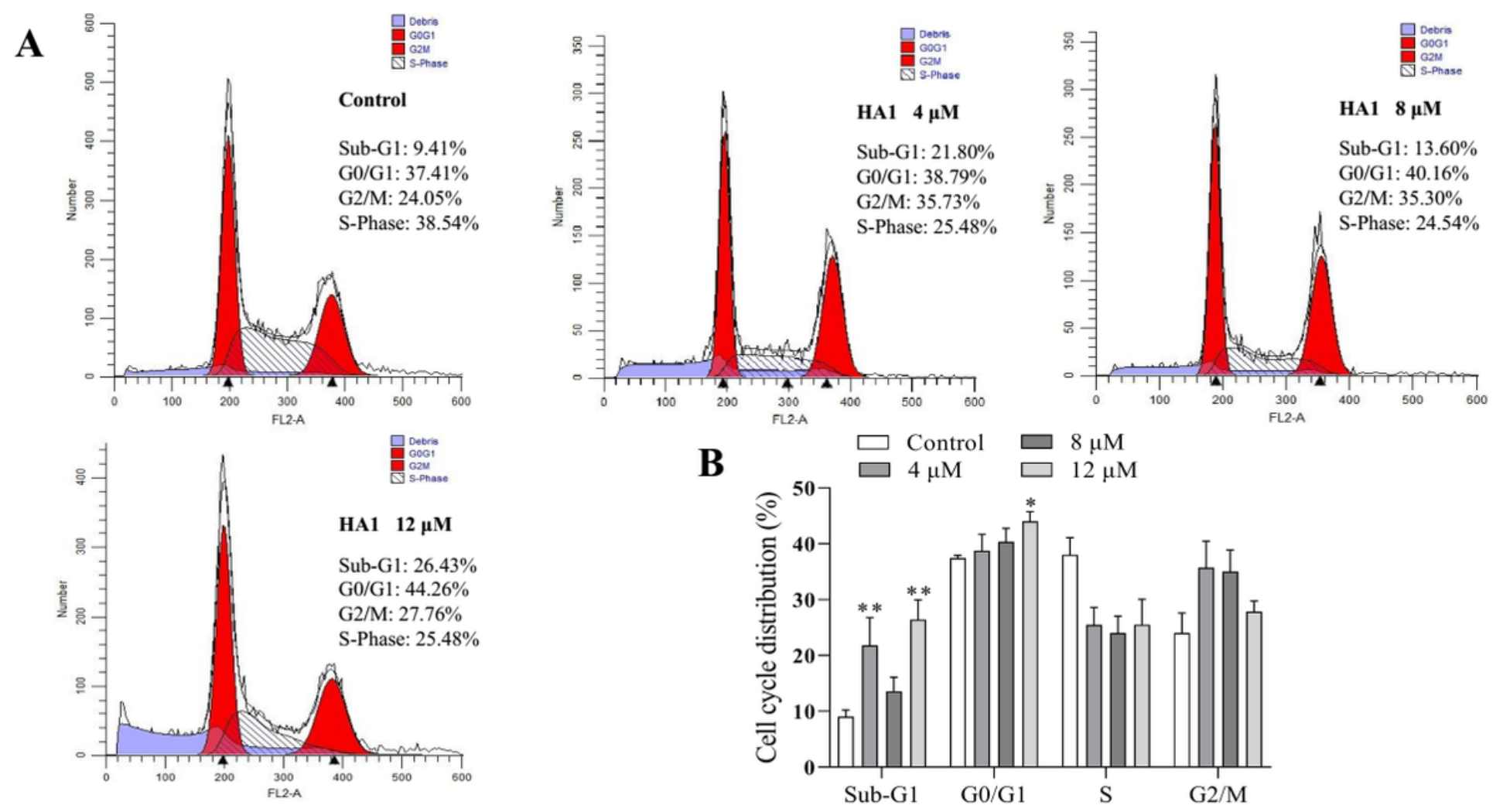

Figure 2

HA1 induced the cell cycle arrest of MCF-7 cells. (A): HA1 significantly inhibited the cell cycle at Sub-G1 and G0/G1 phases after $24 \mathrm{~h}$ incubation. $\left(A^{\prime}\right)$ : The bar chart exhibited the values of mean \pm S.D. of three times. ${ }^{*} \mathrm{P}<0.05$ vs. Control; ${ }^{* *} \mathrm{P}<0.01$ vs. Control.

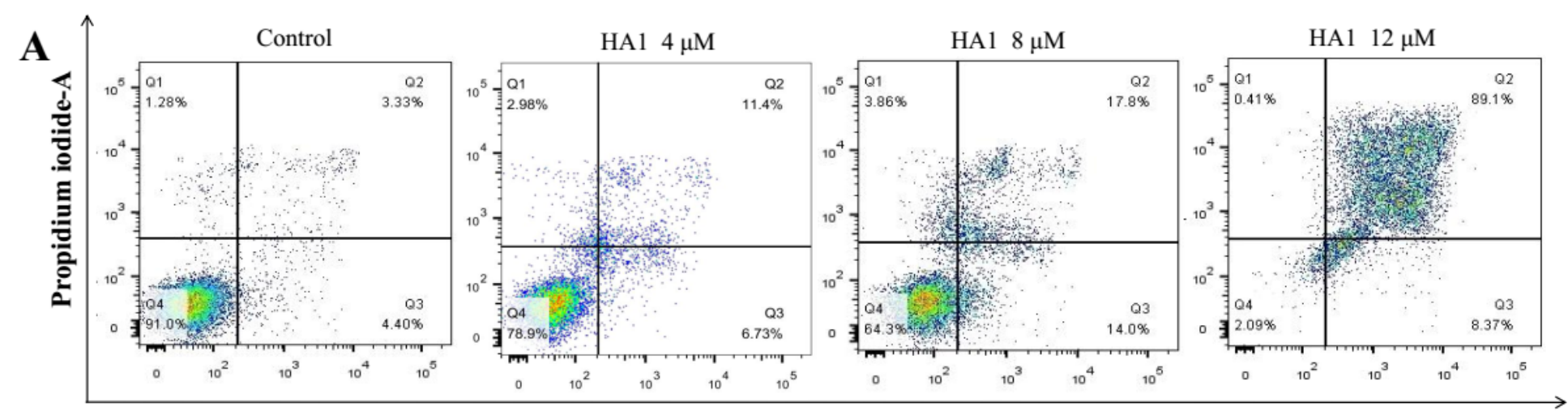

FITC-A

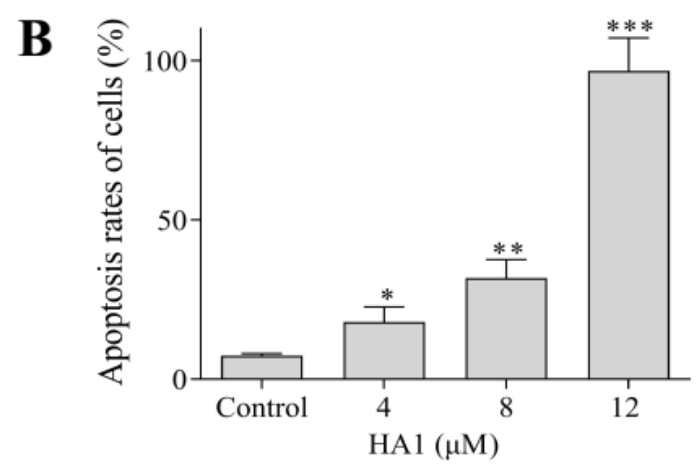

Figure 3 
HA1 induced the apoptotic changes MCF-7 cells. (A): HA1 promoted the raise of apoptosis ratio of MCF-7 cells for $48 \mathrm{~h}$. (B): The bar chart exhibited the values of mean \pm S.D. of three times. ${ }^{*} P<0.05$ vs. Control; ${ }^{*} \mathrm{P}<0.01$ vs. Control; $* * * \mathrm{P}<0.001$ vs. Control.

A
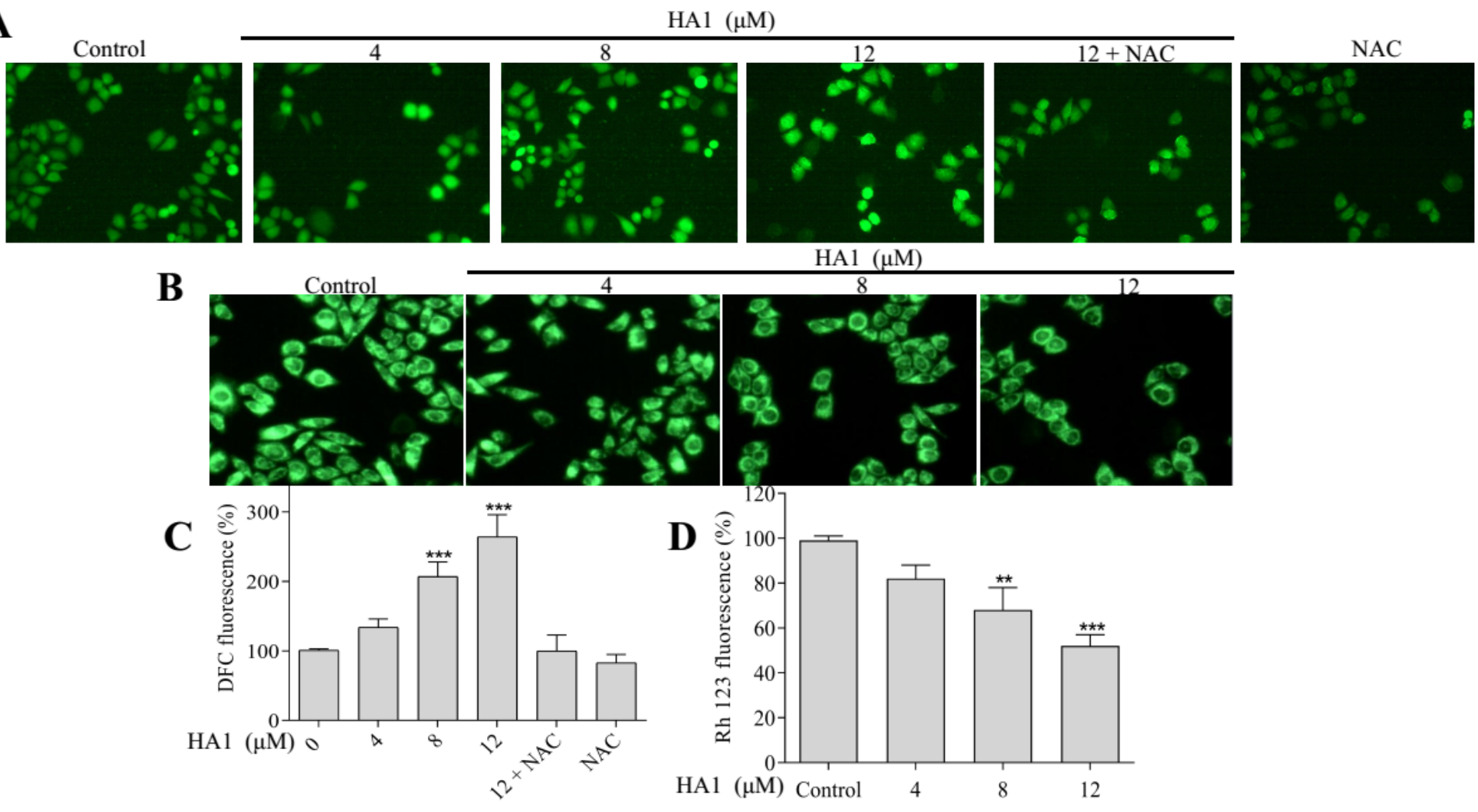

\section{Figure 4}

HA1 increase the levels of ROS and decrease MMP in MCF-7 cells. (A): Microscope results of HA1 reduced the ROS generation after 30 min incubation. (B): Microscope results of HA1 reduced the MMP of MCF-7 cells after $24 \mathrm{~h}$ incubation. (C, D): The bar chart exhibited the values of mean \pm S.D. of three times. **P $<0.01$ vs. Control; *** $\mathrm{P}<0.001$ vs. Control.
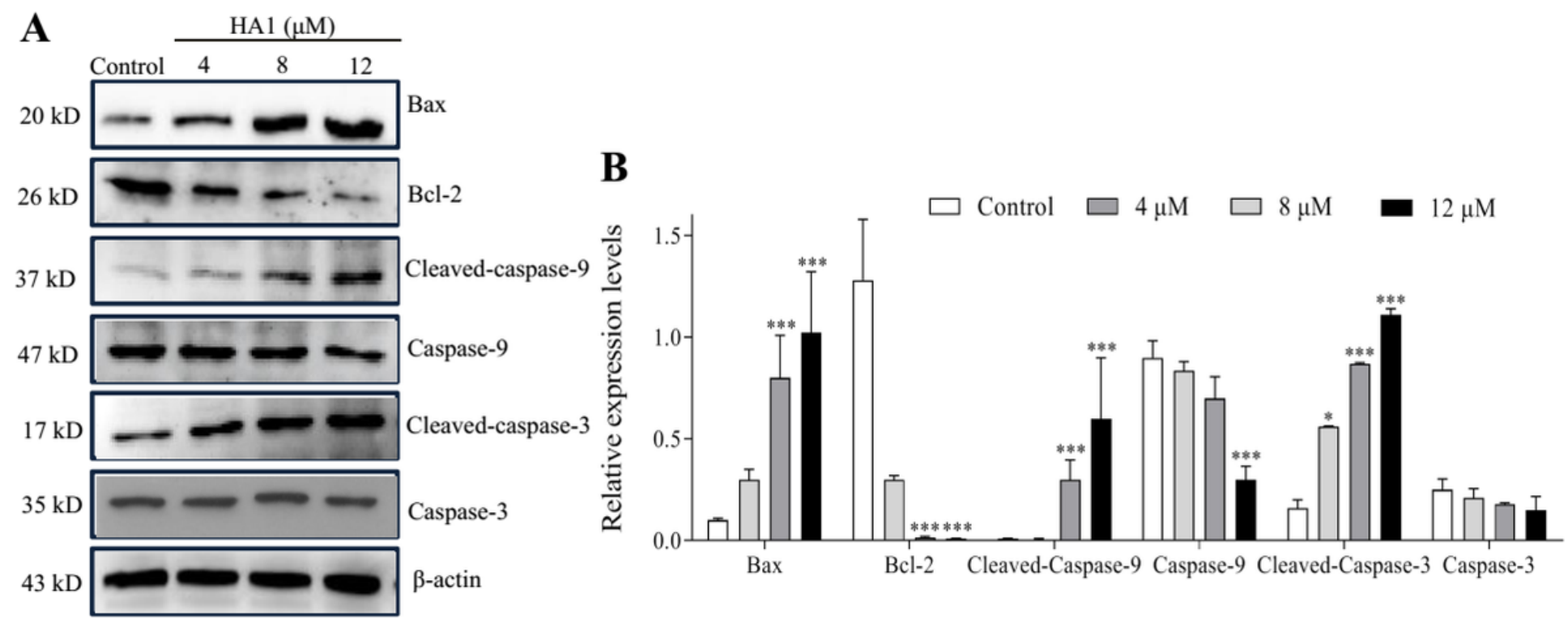

Figure 5 
HA1 induced the apoptotic response of MCF-7 cells by activating mitochondrial pathway. (A): The levels of Bax, Bcl-2, caspase-3/9 and cleaved-caspase-3/9 were significantly decreased by HA1; ( $\left.A^{\prime}\right)$ : The bar chart exhibited the values of mean \pm S.D. of three times. $\beta$-actin was the internal control. ${ }^{*} P<0.05$ vs. Control; $* * \mathrm{P}<0.01$ vs. Control; $* * * \mathrm{P}<0.001$ vs. Control.

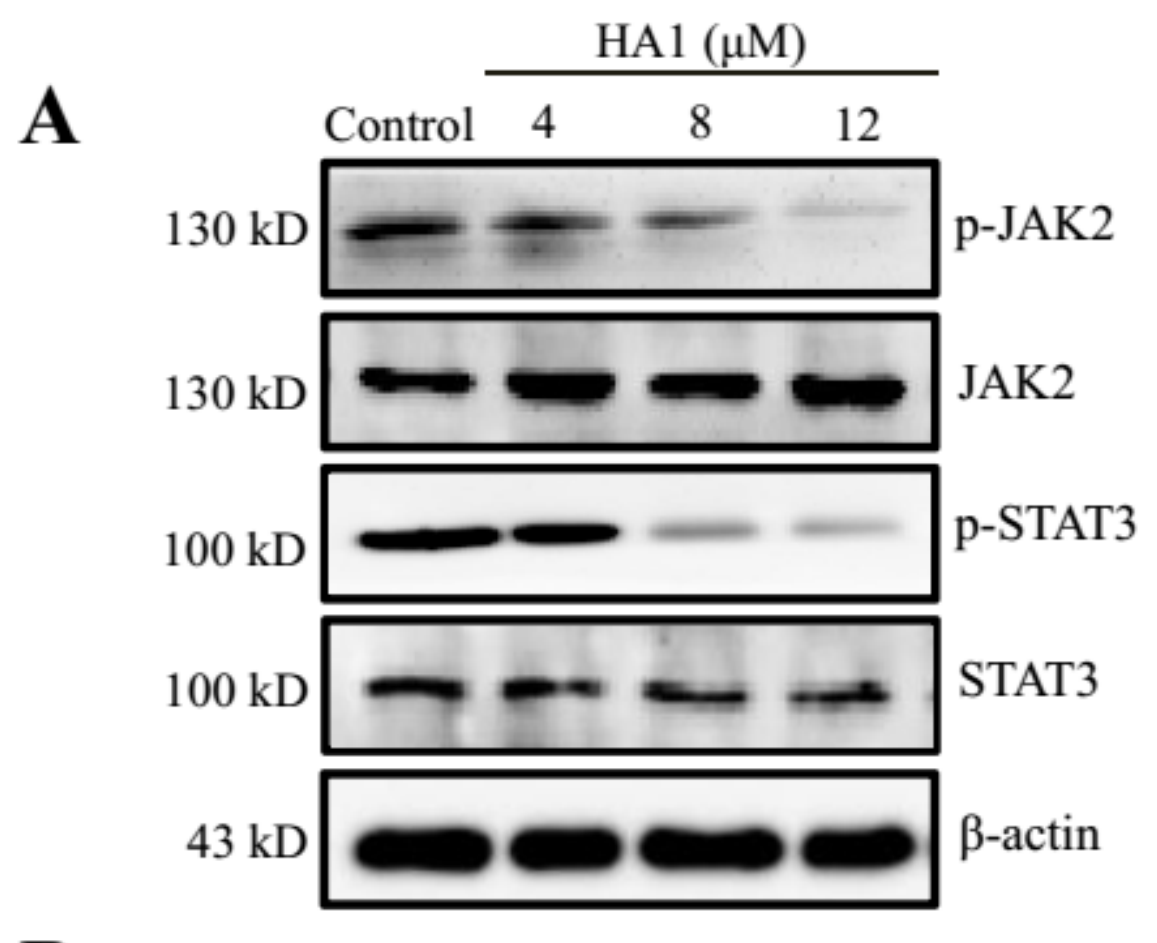

B

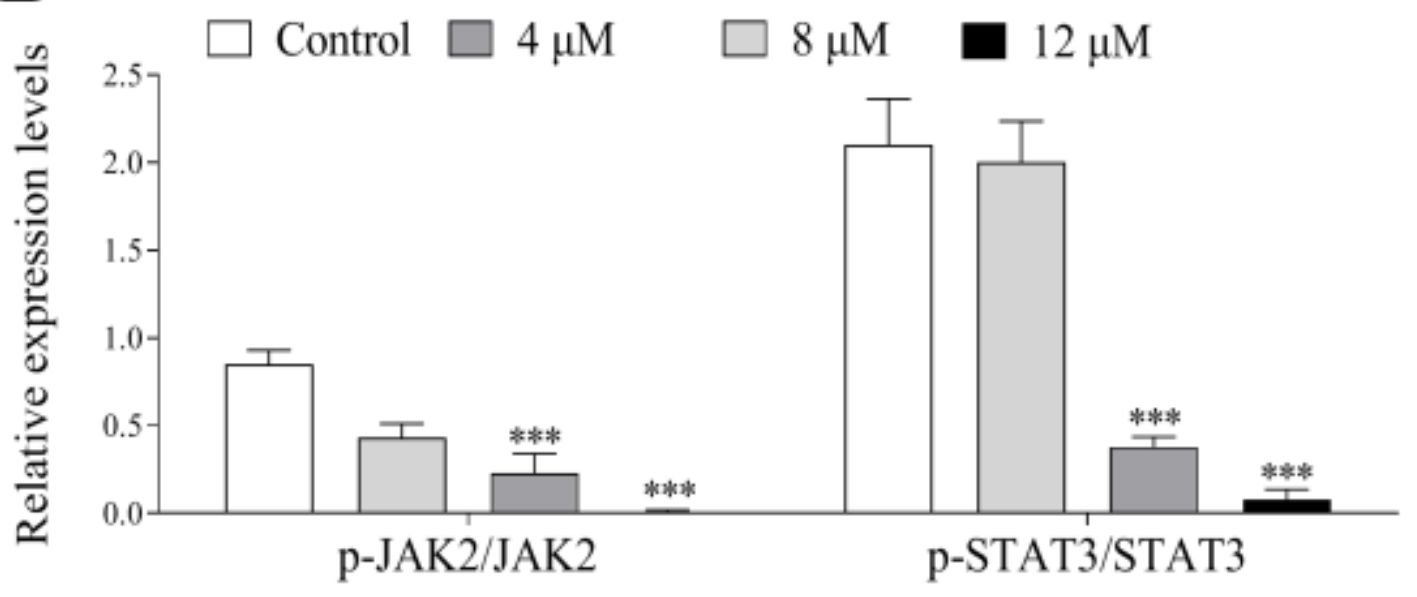

\section{Figure 6}

HA1 inactivated JAK2/STAT3 signaling pathway. (A): HA1 reduced the expression levels of $p-J A K 2$ and pSTAT3 in MCF-7 cells. ( $\left.A^{\prime}\right)$ : The bar chart exhibited the values of mean \pm S.D. of three times. $\beta$-actin was the internal control. ${ }^{*} \mathrm{P}<0.05$ vs. Control; $* * \mathrm{P}<0.01$ vs. Control; $* \star \star P<0.001$ vs. Control. 
A
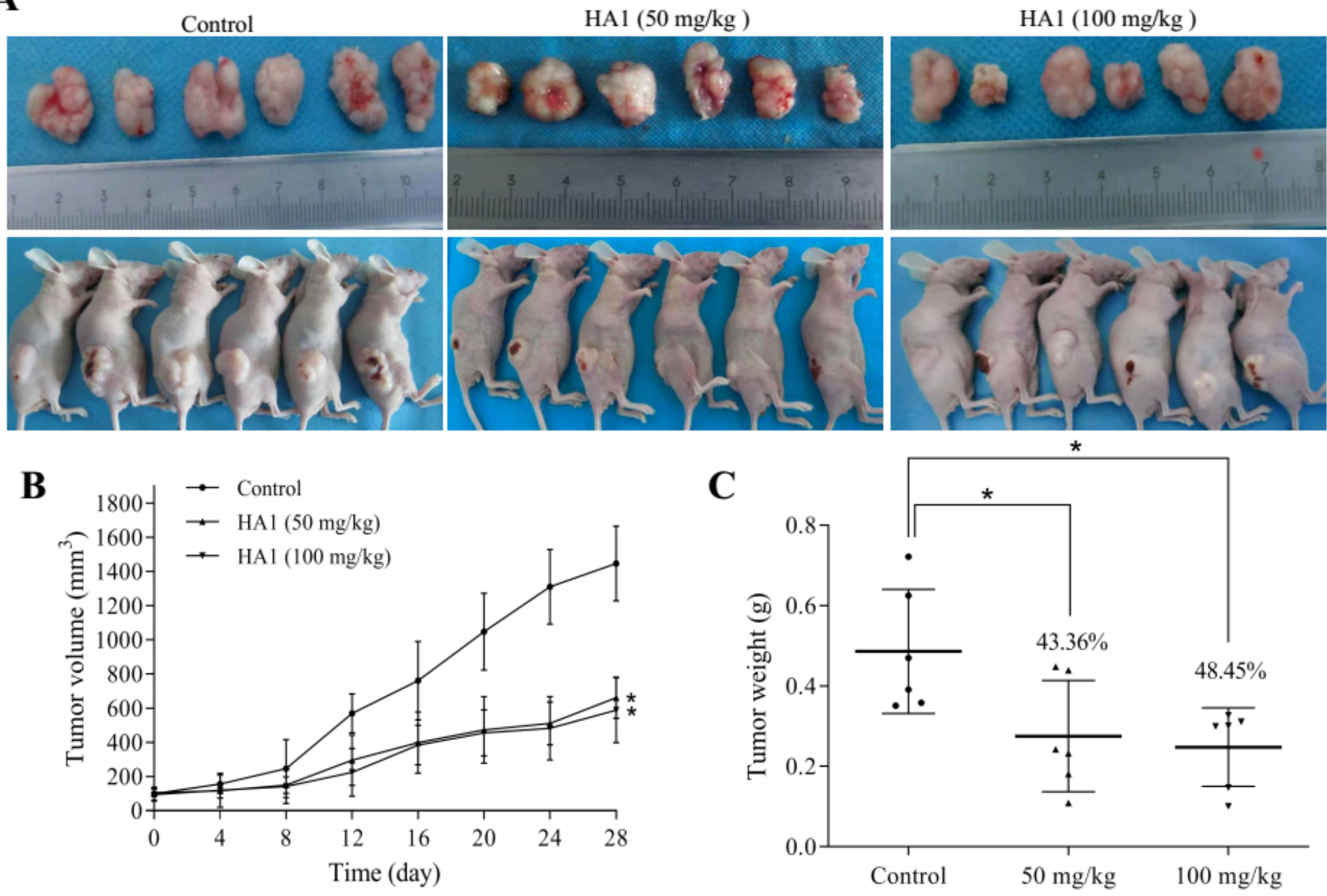

Figure 7

Anti-tumor effects of HA1 on MCF-7 cell xenograft model. (A): The photographs of isolated tumors after the treatment of saline and HA1 for 28 days. HA1 could significantly inhibit the tumor volume (B) and tumor weight (C) after 28 days treatment. There are no significant difference of viscera index (D) and body weights $(E)$ of all groups. Data were expressed as mean \pm S.D. $* P<0.05$ vs. Control.

\section{Supplementary Files}

This is a list of supplementary files associated with this preprint. Click to download.

- STable1.docx 